\title{
INTERURBAN ROAD NETWORK PLANNING MODEL WITH ACCESSIBILITY AND ROBUSTNESS OBJECTIVES
}

\author{
Bruno F. Santos ${ }^{a}$, António P. Antunes ${ }^{*}$ \& Eric J. Miller ${ }^{b}$ \\ ${ }^{a}$ Department of Civil Engineering, University of Coimbra, Portugal \\ ${ }^{b}$ Department of Civil Engineering, University of Toronto, Canada
}

\begin{abstract}
Road network planning (or design) problems consist of determining the best investment decisions to be made with regard to the improvement of a road network. In this paper, we propose an optimization model for long-term interurban road network planning where accessibility and robustness objectives are simultaneously taken into account. Three network robustness measures were defined to assess different robustness concerns: network spare capacity; city evacuation capacity; and network vulnerability. The results that may be obtained from the application of the model are illustrated for three random networks. Special attention is given to the implications of adopting each one of the robustness measures upon the optimum solution provided by the model.
\end{abstract}

Key Words: Road network design; Robustness; Reliability; Optimization model

\footnotetext{
* Corresponding author

Address: Department of Civil Engineering, University of Coimbra, Polo II, 3030-788 Coimbra, Portugal. Telephone: (+351) 239797139. Fax: (+351) 239797142. E-mail: antunes@dec.uc.pt
} 


\section{Introduction}

Road network planning (or design) problems consist of determining the best investment decisions to be made with regard to the improvement of a road network. The degradation of the quality of service provided by the network that may occur in case of fluctuations in travel demand or disruptions in infrastructure supply is typically not taken into account in models designed to represent those problems. Yet, this type of occurrences can have a severe impact on both the welfare of individual drivers and the performance of economic systems as a whole.

The probability and the consequences associated with the failure or malfunctioning of some road network elements became an important topic of research in transportation planning in the 1990's. However, in spite of the increasing interest in developing methodologies to assess the robustness (or reliability) of transportation networks, few studies have focused on the integration of robustness objectives into road network planning models.

The aim of this paper is to propose an optimization model for long-term interurban road network planning where accessibility and robustness objectives are simultaneously taken into account and to discuss the implications of adopting different robustness measures. Three network robustness measures were defined to assess different robustness concerns: network spare capacity; city evacuation capacity; and network vulnerability. The model is intended at helping road authorities in their strategic, system-wide decisions regarding the long-term evolution of an interurban road network. It does not apply when the purpose is to make detailed decisions on which roads to improve among a small number of alternatives.

The paper is organized as follows. We start by introducing several alternative robustness concepts. Next, we present the formulation of the optimization model and the three measures used to assess network robustness. Afterward, we describe the method used to solve the model and analyze the calculation effort associated with its implementation. Then, we discuss and compare the results obtained through the model for three random networks designed to replicate real-world networks. In the final section, we briefly summarize our results and provide some concluding remarks. 


\section{Robustness Concepts}

Robustness is a complex concept. As it often happens with complex concepts, several definitions of robustness are available. One of the best-known is due to Ziha (2000), who defines robustness as the capacity of a system (network) to respond to adverse conditions. The concept of robustness is closely related to the concept of reliability. For Bell and Iida (1997), reliability is the degree of stability of the quality of service which a system normally offers. According to Immers et al. (2004), the distinction between these two concepts resides in the fact that reliability refers to the evaluation of the performance of a system, whereas robustness is a characteristic of the system itself.

The initial studies on network robustness focused on two concepts of reliability. The first, connectivity reliability, considers the probability that two network centers (nodes) remain connected when one or more links are cut (Wakabayashi and Iida 1992, Bell and Iida 1997). The second, travel time reliability, considers the probability of making a trip between two network centers within a specified time interval given daily stochastic travel demand variation (Asakura and Kashiwadani 1991, Clark and Watling 2005). More recently, Chen et al. (1999) introduced the concept of capacity reliability, which is concerned with the probability that a network can accommodate a certain travel demand at a given level of service. Yang et al. (2000) compared capacity reliability and travel time reliability, and suggested that they should be used together on comprehensive road network design.

In the last five years, some authors proposed the concept of vulnerability or potential reliability for dealing with the potential consequences of link failure in addition to the probability of failure (Berdica 2002, D'Este and Taylor 2003). D'Este and Taylor (2003) define vulnerability as being the susceptibility of a network to a significant reduction in accessibility in case of loss (or degradation) of a small number of links. These links should be those whose failure provokes the largest reduction in network performance. An interesting application of this concept to the Australian national road network can be found in Taylor et al. (2006). 


\section{Planning Model}

A considerable research effort has been devoted to road network planning models over the last forty years. The vast majority of these efforts were oriented towards two models: the discrete network design problem (DNDP) model and, especially, the continuous network design problem (CNDP) model. The former focus on the addition of new links to a road network, whereas the latter concentrates on the (continuous) expansion of capacity of existing links. Both models are built around an efficiency objective - typically the maximization of user benefits or the minimization of user costs. Among the best-known articles where these models are dealt with one may quote LeBlanc (1975) and Boyce and Janson (1980) regarding the DNDP model, and Abdulaal and LeBlanc (1979), Leblanc and Boyce (1986), Suwansirikul et al. (1987), and Friesz et al. (1992) regarding the CNDP model. For a relatively recent review of this literature, see Yang and Bell (1998).

The consideration of robustness issues within road network planning models is relatively rare. Most of the existing works where this type of issues is explicitly addressed deal with the CNDP: Sumalee et al. (2006), Ukkusuri et al. (2007), Chen et al. (2007), and Lo and Tung (2003). The former three focus on demand uncertainty and travel time reliability, while the latter focus on capacity reliability when minor disruptions occur. More recently, Chen et al. (2008) considered both travel time and capacity reliability into a bi-objective CNDP, as previously suggested by Yang et al. (2000). To the best of our knowledge, the only article published in scientific journals dealing with robustness issues within the DNDP is Scaparra and Church (2005). In their study, Scaparra and Church considered an all-season connectivity reliability objective for a rural road network where complete coverage by all-season roads was not strictly required.

The model proposed in this paper has a number of important, distinctive features. First, it is a multi-level model. That is, road capacity increases are discrete, as it occurs in practice when, say, a two-lane highway is converted into a four-lane freeway. Despite their obvious practical interest, we are aware of only two articles where multi-level models are dealt with - Janson et al. (1991) and Antunes et al. (2003). Second, it relies on an assignment principle different from the traditional user equilibrium principle. Our 
assignment principle is: "drivers follow the routes that minimize their costs when they travel at an average speed consistent with the design level of service of the links included in the routes". The reasons for adopting this principle are due to the specificities of interurban road traffic, and are thoroughly explained in Santos et al. (2007). Third, it assumes travel demand to be elastic in respect to both trip distribution and traffic induction. That is, both the total volume of traffic generated by (attracted to) some center and the distribution of this traffic by the other centers depend on the future connections serving the center. Fourth and last, it is a bi-objective model combining an efficiency objective with a robustness objective. The efficiency objective considered is the maximization of accessibility. For assessing accessibility, we use an indicator based on the well-known Hansen local accessibility measure (Hansen 1959). This measure has been used in numerous studies, either for analyzing accessibility issues in large regional areas (see e.g. Keeble et al. 1982, Vickerman et al. 1999, and Paez 2004) or for evaluating the accessibility implications of major transportation infrastructures (see e.g. Vickerman 1987, Jensen-Butler and Madsen 1996, and Gutiérrez 2001).

The mathematical formulation of the model is as follows:

$$
\max Z=w_{A} \times \frac{A-A_{0}}{A_{B}-A_{0}}+w_{R} \times \frac{R-R_{0}}{R_{B}-R_{0}}
$$

subject to

$$
\begin{aligned}
& A=\sum_{j \in N} P_{j} \times \sum_{k \in N \backslash j} \frac{P_{k}}{C_{j k}(y)^{\beta}} \\
& R=\phi(y) \\
& \sum_{m \in M_{l}} y_{l m}=1, \quad \forall l \in \boldsymbol{L} \\
& T_{l}(\boldsymbol{y}) \leq \sum_{m \in M_{l}} F_{m} \times y_{l m}, \quad \forall l \in \boldsymbol{L} \\
& \sum_{l \in L} \sum_{m \in M_{l}} e_{l m} \times y_{l m} \leq b \\
& T_{l} \geq 0, y_{l m} \in\{0,1\}, \forall l \in \mathbf{L}, m \in \boldsymbol{M}_{l}
\end{aligned}
$$


where $Z$ is the normalized value of a solution; $w_{A}$ and $w_{R}$ are the weights attached to the accessibility and robustness objectives, respectively; $A$ and $R$ are the values of a solution in terms of each objective; $A_{B}$ and $A_{0}\left(R_{B}\right.$ and $\left.R_{0}\right)$ are reference maximum and minimum values for each objective; $\boldsymbol{N}$ is the set of centers; $P_{j}$ is the population of center $j$ (or any other measure of the size of the center); $C_{j k}$ is the generalized cost for traveling between centers $j$ and $k ; \boldsymbol{y}=\left\{y_{l m}\right\}$ is a matrix of binary variables equal to one if link $l$ is set at road type $m$ and equal to zero otherwise; $\boldsymbol{M}_{l}$ is the set of possible road types for link $l ; \boldsymbol{L}$ is the set of links; $T_{l}$ is the estimated traffic flow in link $l ; F_{m}$ is the maximum service flow for a link of road type $m$; $e_{l m}$ is the expenditure required to set link $l$ at road type $m$; and $b$ is the budget.

The (main) decision variables of this non-linear combinatorial optimization model are the $\boldsymbol{y}$ variables, which represent the road type to assign to the various links of the network. The objective-function (1) expresses the normalized value of model solutions considering both the accessibility objective and the robustness objective. Weights are applied to the objectives to reflect their relative importance. Constraint (2) defines the accessibility measure (as a weighted sum of Hansen local accessibilities). Constraint (3) defines the robustness measure as being dependent on the road type assigned to the various links of the network. Several alternatives for the robustness measure are presented in the next section. Constraints (4) guarantee that each link will be set at one, and only one, road type. For some links, it may be undesirable to choose some road types because of environmental reasons. This is the reason why the set of road types $\left(\boldsymbol{M}_{l}\right)$ is indexed in the link. Constraints (5) ensure that the traffic flow estimated for each link will not exceed the maximum service flow consistent with the road type chosen for the link. Constraint (6) guarantees that the budget available for improving the network will not be exceeded. Finally, expression (7) defines the domain for the decision variables.

\section{Robustness Measures}

As mentioned above, there are several robustness concepts applicable to road network planning. For our model, we decided to address robustness through three reliability measures, two of them of the capacity reliability type and the other of the vulnerability type. The measures are presented below. 


\subsection{Network spare capacity}

When planning an interurban road network it is difficult to predict the travel demand and the infrastructure supply variations that will occur throughout its lifetime. The lack of capability of the network to respond to these variations can lead not only to serious local disturbances but also to the propagation of the disturbances across the network. A good way of giving this capability to the network is to provide links with spare capacity to accommodate the supply or demand variations. In order to measure the overall spare capacity available in the network, we defined the following network spare capacity index:

$$
S(\boldsymbol{y})=\frac{\sum_{l \in \boldsymbol{L}}\left[F_{l}(\boldsymbol{y})-T_{l}(\boldsymbol{y})\right]^{\alpha} \times T_{l}(\boldsymbol{y}) \times L_{l}}{\sum_{l \in \boldsymbol{L}} T_{l}(\boldsymbol{y}) \times L_{l}}
$$

where $F_{l}$ is the maximum service flow for link $l$ (given its type); $\alpha$ is a weighting parameter; and $L_{l}$ is the length of the link $l$.

This index is calculated by summing the spare capacity of each link (affected by parameter $\alpha$ ) weighted by the total number of kilometers traveled on the link (vehicles $\times \mathrm{km}$ ) and dividing the sum with the total number of kilometers traveled on the network. Weights are applied to emphasize the importance of spare capacity in long links with large traffic flows. The parameter $\alpha$ is introduced to reflect the importance attached to the spare capacity in each link. Values of $\alpha$ higher than one lead to solutions where spare capacities are large but concentrated on a small number of links, whereas values of $\alpha$ lower than one lead to solutions where spare capacities are relatively small but more evenly distributed across the network.

\subsection{City evacuation capacity}

Situations of localized high travel demand can be critical for the performance of an interurban road network. The capability of the network to allow the fast evacuation of cities can be essential for public safety in case of unexpected occurrences (e.g., terrorist attacks, weather calamities) or for the mobility of the visitors of major planned occurrences (e.g., music festivals, sport events). This subject has become extremely important in recent years, after the last terrorist attacks and Hurricane Katrina. In order 
to represent the aptitude of a network for dealing with this kind of circumstances, we defined the following city evacuation capacity index:

$$
X(y)=\frac{\sum_{j \in N} \sum_{l \in F(j)} Q_{l}(y) \times P_{j}}{P}
$$

where $\boldsymbol{F}(j)$ is the forward star for center $j$ (i.e., the links with origin in center $j$ ), $Q_{l}$ is the maximum flow capacity of link $l$, and $P$ is the total population of the region.

The index is calculated by summing the total capacity of the links with origin in each city weighted by the population of the city, and dividing the sum by the total population of the region. Weights are applied to emphasize the importance of evacuation capacity in large cities.

\subsection{Network vulnerability}

The failure of some links can have serious consequences on the overall performance of an interurban road network, particularly when the network is sparse (which is often the case). A network that is less vulnerable to the failure of links is a network that is more robust, and vice-versa (Berdica 2002). In order to reduce the vulnerability of a network, these potential consequences must be mitigated even when the probability of link failure is low. For representing the vulnerability of a network, we defined the following index:

$$
V(y)=\sum_{l \in N_{p}} \frac{\sum_{j \in N} \sum_{k \in N} C_{j k}^{l}(y) \times T_{j k}(y)-\sum_{j \in N} \sum_{k \in N} C_{j k}(y) \times T_{j k}(y)}{N_{p}}
$$

where $N_{p}$ is the set of links with the $p$ largest vulnerability indexes; $C_{j k}^{l}$ is the generalized cost for traveling between centers $j$ and $k$ when link $l$ is closed; $T_{j k}$ is the traffic flow between centers $j$ and $k$; and $N_{p}$ is the number of links in the $N_{p}$ set.

Expression (10) defines network vulnerability as the average increase on travel costs when the links with the largest vulnerability indexes are closed separately. Link vulnerability indexes $\left(v_{l}\right)$ are defined taking two aspects into account: the number of least-cost routes where the link is included; and the traffic flow on the link. These two aspects are weighted according to their importance. The expression used for the calculation of the link vulnerability indexes is as follows: 


$$
v_{l}=\left\{\begin{array}{c}
0, \forall l \in \boldsymbol{L}_{U} \\
w_{n} \times \frac{n_{l}(\boldsymbol{y})-n_{\min }}{n_{\max }-n_{\min }}+w_{T} \times \frac{T_{l}(\boldsymbol{y})-T_{\min }}{T_{\max }-T_{\min }}, \forall l \in \boldsymbol{L} \backslash \boldsymbol{L}_{U}
\end{array}\right.
$$

where $\boldsymbol{L}_{U}$ is the set of links which cannot be closed; $w_{n}$ is the weight attached to inclusion in least-cost routes; $n_{l}$ is the number of least-cost routes where link $l$ is included; $n_{\max }\left(n_{\min }\right)$ is the maximum (minimum) number of least-cost routes where the same link is included; $w_{t}$ is the weight attached to traffic flow; $T_{\max }\left(T_{\min }\right)$ is the maximum (minimum) traffic flow on a link.

The links in the $\boldsymbol{L}_{U}$ set cannot be closed because some centers would not be accessible from some other centers; that is, these are the links of the only possible route connecting a center, or a set of centers, to the other centers. Since there are no alternatives to these links, the vulnerability associated with them cannot be mitigated. Therefore, they were excluded from the analysis (by assuming their vulnerability index to be zero).

\section{Solution Method}

The optimization model previously described is extremely difficult to solve. In general, it can only be solved to exact optimality for small instances (up to ten centers and twenty links), through an exhaustive enumeration approach. For larger instances, it is necessary to resort to heuristic methods. The heuristics available before the 1980s often led to local optimum solutions sometimes quite far from a global optimum. Since then, a large number of modern heuristics have been devised, including genetic algorithms, tabu search, simulated annealing, and cross-entropy algorithms (Michalewicz and Fogel 2004, Gendreau and Potvin 2005). These heuristics often avoid poor local optimum solutions and find either a global optimum or a very good local optimum.

For solving the model we developed an Enhanced Genetic Algorithm (EGA). This algorithm improves on the classic genetic algorithm in several respects (Figure 1). First, it includes local search (add and drop) procedures in order to repair solutions which do not take full advantage of the budget available or do not comply with it. Second, it uses interchange procedures for the best solution found after a given number of iterations and for the best solution found immediately before ending the algorithm. Third, it utilizes an 
intervention procedure after a given number of iterations, through which the parameters governing the selection, crossover, mutation, and invasion operations may change slightly.

\section{(Locate Figure 1 approximately here)}

The EGA was chosen to solve the model after comparing its performance with the performance of two other algorithms, namely an Add \& Interchange algorithm and a Variable Neighborhood Search algorithm. The comparison was made for a sample of 80 random networks of 20 to 100 nodes (and 30 to 220 links) designed to mimic real-world networks, considering only the efficiency objective $\left(w_{A}=1\right.$ and $\left.w_{R}=0\right)$. When applied to large networks the EGA clearly outperformed the other algorithms (Santos et al. 2005). Furthermore, when applied to small networks for which we were able to find optimum solutions through exhaustive enumeration only after several hours of computation, the EGA was the only algorithm that was always able to identify a global optimum solution requiring for this no more than a few seconds.

The computing time involved on the application of the EGA to models considering efficiency and robustness objectives was studied on a sample of 20 random networks, also of 20 to 100 centers, with weights for the objectives varying from 10 to 90 percent. The study was made using regression analysis and has shown that there is no dependence between computing time and the weights assigned to the objectives ( $t$-tests on the regression coefficients clearly below 2.0). It has also shown that, depending on the robustness measure, computing time grows with instance size according to the following power functions:

- network spare capacity: $T=4.412 \times 10^{-6} \times N^{3.565}\left(R^{2}=0.971\right)$

- city evacuation capacity: $T=8.148 \times 10^{-6} \times N^{3.398}\left(R^{2}=0.952\right)$

- network vulnerability: $T=2.183 \times 10^{-3} \times N^{2.967}\left(R^{2}=0.757\right)$

where $T$ is the computing time in minutes and $N$ is the number of network links.

The computing effort involved in the utilization of the vulnerability index is much larger than the computing effort required when the other two robustness measures are used. According to the above expressions, the expected computing time needed to solve 
100- and 200-link instances with a Dual Core computer running at 2.6 GHz is 59.5 minutes and 11.7 hours when the measure is network spare capacity, and to 50.9 minutes and 8.9 hours when the measure is city evacuation capacity. When the robustness measure is network vulnerability the computing time increases to 31.5 hours and to 10 days, respectively. That is, computing time becomes much higher. Note, however, that they were obtained when 20 percent of the network links were considered for the analysis of vulnerability ( $N_{p}=0.2 \times L$, where $L$ is the number of links). Lower values of $\mathrm{Np}$ would considerably reduce the computation burden. For instance, with $N p=0.05 \times L$, the computing time reduces to 12.9 hours and 4 days, for the 100 - and 200-link instances.

\section{Application Examples}

The type of results that can be obtained through the model and, especially, the impact of robustness concerns upon optimum solutions, are exemplified below for three random road networks (generated with the same rules as the networks used to analyze the computing times required for solving the model). The three networks are depicted in Figure 2. They consist of 10 population centers, several existing links and a few possible links. The centers were named according to their population rank: center 1 is the largest center; center 2 is the second-largest; and so forth. Existing roads types are slow two-lane highway (free-flow speed equal to $70 \mathrm{~km} / \mathrm{h}$ ) and fast two-lane highway (90 km/h). Two other road types were defined for possible link construction or upgrading: four- and six-lane freeway (both $120 \mathrm{~km} / \mathrm{h}$ ). The costs per kilometer for road construction and upgrading are presented in Table I. The budget available for the improvement of each network was set equal to 30 percent of the total expenditure involved in upgrading all links to a six-lane freeway.

(Locate Figure 2 approximately here)

(Locate Table I approximately here)

The model was first applied to the networks considering only the accessibilitymaximization objective. Then, the robustness objective was added to the model, giving both objectives the same weight (50/100) and considering the three measures introduced 
above one at a time. In the computation of network spare capacity we used $\alpha=0.2$, and in the computation of network vulnerability we used $w_{n}=0.3, w_{t}=0.7$, and $N_{p}=0.2 \times L$, where $L$ is the number of links.

\section{Results}

\subsection{Accessibility Maximization}

When only the accessibility-maximization objective is considered, the optimum solution consists mainly of improving links connecting large centers, with the remaining budget being assigned to the upgrade of links connecting either large centers to neighboring small centers or two neighboring small centers with each other (Figure 3). For example, in Network 1, the two largest centers are connected with each other and to their neighboring centers by four-lane freeways. The link which connects these freeways with remote Center 4 is improved to a fast two-lane highway. For Network 2, all links starting at Center 1 are upgraded to two-lane freeways. For Network 3, the optimum solution is of a different nature, because the largest centers are far away from each other. Centers 2 and 3 are connected by four-lane freeway, and the same occurs for the cluster formed by Centers 5, 6, 7, and 10. In this case Center 1 is not linked to the other large centers by freeway, but is linked to the cluster of centers by a fast two-lane highway and to the closest center (Center 9) by four-lane freeway. With regard to the initial situation, the increase in accessibility is $47.7,34.3$, and 35.3 percent for Networks 1, 2, and 3, respectively (Table II).

(Locate Figure 3 approximately here)

(Locate Table II approximately here)

\subsection{Network spare capacity}

When network spare capacity is included as the robustness objective, the optimum solutions involve, in general, the upgrade of the links with higher traffic flows to sixlane freeways (Figure 4). These links are essentially the links connecting Center 1 to other large centers or to neighboring centers. Since six-lane freeway is the most expensive road type, the budget constraint prevents the upgrade of other links, thus reducing the accessibility of both small centers and centers located far away from 
Center 1. In comparison with the maximum accessibility solutions, Center 4 in Network 1 is not served by a fast road and Centers 9 and 10 in Network 2 are not connected by freeway to Center 1 . In Network 3, Center 1 is now linked through a six-lane freeway with Centers 2 and 3, whereas the connection of the cluster formed by Centers 5, 6, 7, and 10 with Center 1 has not changed (remains a slow two-lane highway). With regard to the initial situation, the increase in spare network capacity is 19.9, 14.3, and 13.5 percent for Networks 1, 2, and 3, respectively, while accessibility increased only 45.1, 30.5, and 30.4 percent. As one could expect, these accessibility increases are smaller than the increases achieved when the only objective is to maximize accessibility.

(Locate Figure 4 approximately here)

\subsection{City evacuation capacity}

When network spare capacity is replaced with city evacuation capacity, the optimum solutions involve in general the improvement of the links leaving from the largest centers (Figure 5). For both Network 1 and Network 3, a significant part of the budget is used to upgrade all links connected to Center 1, often to six-lane freeways. The remaining budget is assigned to the upgrade of small links or links leaving from other large centers. For Network 2, not all of the links connected to Center 1 are upgraded. In this case, the upgrade of the longest link with origin at Center 3 is not made because of budget limitations, while the other links leaving Center 1 are upgraded to the link type with maximum capacity. With regard to the initial situation, the increase in city evacuation capacity is 258.8, 152.9, and 181.5 percent for Networks 1, 2, and 3, respectively, while accessibility increased 46.7, 30.3, and 32.3 percent.

(Locate Figure 5 approximately here)

\subsection{Network vulnerability}

When city evacuation capacity is replaced with network vulnerability, the optimum solutions involve in general the upgrading of links which can constitute alternative routes to travel between the largest centers and to centers with poor connections (Figure 6). For Network 1, a new link is built between Centers 2 and 10, to provide a second good connection between Centers 1 and 2. Also, Center 10 has now more than one connection to the rest of the network. For Network 2, Centers 4, 7, and 10 originally had 
only one connection to the network. The first two centers now have a second connection, whereas the third, due to its small population, keeps the only connection it had before. With regard to the initial situation, the decrease in network vulnerability is 75.5, 82.7, and 78.2 percent for Networks 1, 2, and 3, respectively, while accessibility increased 46.9, 33.5, and 34.5 percent.

(Locate Figure 6 approximately here)

\subsection{Cross comparisons}

The analysis of the results presented before clearly reveals that the optimization of the robustness of a network according to some robustness measure does not necessarily mean that the network is optimized with regard to the other robustness measures.

In this respect, it is worth noting here that the maximization of accessibility has always been accompanied with the increase of network vulnerability, whereas, at the same time, the spare capacity and the evacuation capacity were improved. In contrast, concern with the minimization of vulnerability has always led to improvements in accessibility, as well as to improvements in spare capacity and evacuation capacity. The accessibility-vulnerability relationship is asymmetric because these two objectives lead to different applications of the budget. When the only objective is to maximize accessibility, the budget is mostly assigned to upgrading the least cost route connecting the largest centers to freeways. As weight is placed on network vulnerability, the budget is also allocated to links on routes that can be an alternative to the least cost route. This amounts to improving a larger set of links, fewer links being upgraded to freeways.

The results obtained for the three networks also revealed that spare capacity and evacuation capacity are closely related; that is, when the objective was to maximize spare capacity the networks were quite good from the evacuation capacity standpoint, and vice-versa. This happens because, in general, the links close to the largest cities are also the links with the larger traffic volumes, hence the links where spare capacity is more necessary. 


\section{Conclusion}

In this paper, we presented an optimization model for long-term interurban road network planning where accessibility and robustness objectives are taken into account. Recent events - such as September 11 and Hurricane Katrina - have drawn the attention of governments and the general public to the importance of including robustness among the objectives to be seriously taken into account when designing infrastructure. For assessing robustness we used three different measures: network spare capacity; city evacuation capacity; and network vulnerability. To our knowledge, this is one of the few papers where both objectives are considered together in a road network planning (or design) model and the only one where a comparison of robustness measures is provided.

The optimization model is quite difficult to solve because of its combinatorial and nonlinear nature. Upon detailed investigation of several possible heuristic approaches, we concluded that the best results are provided by a genetic algorithm enhanced with local search procedures. When network spare capacity and city evacuation capacity are used as robustness measures, the algorithm is expected to solve 200-link instances within 12 hours (on a Dual Core computer running at $2.6 \mathrm{GHz}$ ). Since the model is intended for application at the strategic level of analysis, most real-world road network planning problems will be appropriately represented with networks of this size or less and solved within acceptable computing time. The effort is much more intense when the robustness measure is network vulnerability. In this case, the average time for solving a 200-link problem is approximately 10 days.

The potential usefulness of the model was illustrated for three random networks. In particular, it was shown that the results obtained with or without robustness objectives can vary considerably, even for networks of only ten centers. It was also shown that results largely depend on the measure used for assessing robustness. These are important issues that need to be taken into account in the complex decision-making processes within which interurban road network plans are defined or modified. 


\section{Acknowledgments}

The participation of the first author in the study reported in this article has been supported by Fundação para a Ciência $e$ Tecnologia through grant SFRH/BD/16407/2004.

\section{References}

Abdulaal, M. and Leblanc, L.J., 1979. Continuous equilibrium network design models. Transportation Research Part B-Methodological, 13 (1), 19-32.

Antunes, A., Seco, A. and Pinto, N., 2003. An accessibility-maximization approach to road network planning. Computer-Aided Civil and Infrastructure Engineering, 18 (3), 224-240.

Asakura, Y. and Kashiwadani, M., 1991. Road network reliability caused by daily fluctuation of traffic flow. In: Proceedings of the 19th PTRC Summer Annual Meeting (Seminar G). Brighton, UK, 73-84.

Bell, M.G.H. and Iida, Y., 1997. Transportation network analysis. Chichester, New York, USA: J. Wiley.

Berdica, K., 2002. An introduction to road vulnerability: What has been done, is done and should be done. Transport Policy, 9 (2), 117-127.

Boyce, D.E. and Janson, B.N., 1980. A discrete transportation network design problem with combined trip distribution and assignment. Transportation Research Part BMethodological, 14 (1-2), 147-154.

Chen, A., Kim, J., Zhou, Z. and Chootinan, P., 2007. Alpha reliable network design problem. Transportation Research Record, (2029), 49-57.

Chen, A., Yang, H., Lo, H.K. and Tang, W.H., 1999. A capacity related reliability for transportation networks. Journal of Advanced Transportation, 33 (2), 183-200. 
Chen, A., Zhou, Z., Chootinan, P. and Wong, S.C., 2008. A bi-objective reliable network design problem. In: Proceedings of the 87th Annual Meeting of the Transportation Research Board. Washington DC, USA.

Clark, S. and Watling, D., 2005. Modelling network travel time reliability under stochastic demand. Transportation Research Part B-Methodological, 39 (2), 119-140.

D'Este, G.M. and Taylor, M.A.P., 2003. Network vulnerability: An approach to reliability analysis at the level of national strategic transport networks. In: M. G. H. Bell and Y. Iida, ed. The network reliability of transport. Oxford, UK: Elsevier, 23-44.

Friesz, T.L., Hsun-jung, C., Mehta, N.J., Tobin, R.L. and Anandalingam, G., 1992. A simulated annealing approach to the network design problem with variational inequality constraints. Transportation Science, 26 (1), 18-26.

Gendreau, M. and Potvin, J.Y., 2005. Metaheuristics in combinatorial optimization. Annals of Operations Research, 140 (1), 189-213.

Gutiérrez, G.J., 2001. Location, economic potential and daily accessibility: An analysis of accessibility impact of the high-speed line Madrid-Barcelona-French Border. Journal of Transport Geography, 9 (4), 229-242.

Hansen, W., 1959. How accessibility shapes land use. Journal of the American Institute of Planners, 25, 73-6.

Immers, L.H., Stada, J.E., Yperman, I. and Bleukx, A., 2004. Robustness and resilience of transportation networks. In: Proceedings of the 9th International Scientific Conference MOBILITA. Bratislava, Slovenia.

Janson, B.N., Buckels, L.S. and Peterson, B.E., 1991. Network design programming of united-states highway improvements. Journal of Transportation Engineering-ASCE, 117 (4), 457-478.

Jensen-Butler, C. and Madsen, B., 1996. Modelling the regional economic effects of the danish great belt link. Papers in Regional Science, 75 (1), 1-21. 
Keeble, D., Owens, P.L. and Thompson, C., 1982. Regional accessibility and economicpotential in the european-community. Regional Studies, 16 (6), 419-431.

Leblanc, L.J., 1975. Algorithm for the discrete network design problem. Transportation Science, 39 (1), 63-79.

Leblanc, L.J. and Boyce, D.E., 1986. A bilevel programming algorithm for exact solution of the network design problem with user-optimal flows. Transportation Research Part B-Methodological, 20 (3), 259-265.

Lo, H.K. and Tung, Y.K., 2003. Network with degradable links: Capacity analysis and design. Transportation Research Part B-Methodological, 37 (4), 345-363.

Michalewicz, Z. and Fogel, D.B., 2004. How to solve it: Modern heuristics. Berlin, Germany: Springer-Verlag.

Paez, A., 2004. Network accessibility and the spatial distribution of economic activity in eastern asia. Urban Studies, 41 (11), 2211-2230.

Santos, B., Antunes, A. and Miller, E., 2005. Solving an accessibility-maximization road network design model: A comparison of heuristics. In: A. Jaszkiewicz, M. Kaczmarek, J. Zak, and M. Kubiak, ed. Advanced OR and AI Methods in Transportation. Poznan, Poland, 692-697.

Santos, B., Antunes, A. and Miller, E.J., 2007. A multi-objective approach to road network planning. In: Proceedings of the 11th World Conference on Transportation Research. Berkeley, CA.

Scaparra, M.P. and Church, R.L., 2005. A grasp and path relinking heuristic for rural road network development. Journal of Heuristics, 11 (1), 89-108.

Sumalee, A., Watling, D.P., and Nakayama, S., 2006. Reliable network design problem - case with uncertain demand and total travel time reliability. Transportation Research Record (1964), 81-90. 
Suwansirikul, C., Friesz, T.L. and Tobin, R.L., 1987. Equilibrium decomposed optimization: A heuristic for the continuous equilibrium network design problems. Transportation Science, 21 (4), 254-263.

Taylor, M.A.P., Sekhar, S.V.C. and D'Este, G.M., 2006. Application of accessibility based methods for vulnerability analysis of strategic road networks. Networks \& Spatial Economics, 6 (3-4), 267-291.

Ukkusuri, S.V., Mathew, T.V. and Waller, S.T., 2007. Robust transportation network design under demand uncertainty. Computer-Aided Civil and Infrastructure Engineering, 22 (1), 6-18.

Vickerman, R., 1987. The channel tunnel - consequences for regional growth and development. Regional Studies, 21 (3), 187-197.

Vickerman, R., Spiekermann, K. and Wegener, M., 1999. Accessibility and economic development in europe. Regional Studies, 33 (1), 1-15.

Wakabayashi, H. and Iida, Y., 1992. Upper and lower bounds of terminal reliability of road networks: An efficient method with boolean algebra. Journal of Natural Disaster Science, 14, 22-44.

Yang, H. and Bell, M.G.H., 1998. Models and algorithms for road network design: A review and some new developments. Transport Reviews, 18 (3), 257-278.

Yang, H., Bell, M.G.H. and Meng, Q., 2000. Modeling the capacity and level of service of urban transportation networks. Transportation Research Part B-Methodological, 34 (4), 255-275.

Ziha, K., 2000. Redundancy and robustness of systems of events. Probabilistic Engineering Mechanics, 15 (4), 347-357. 


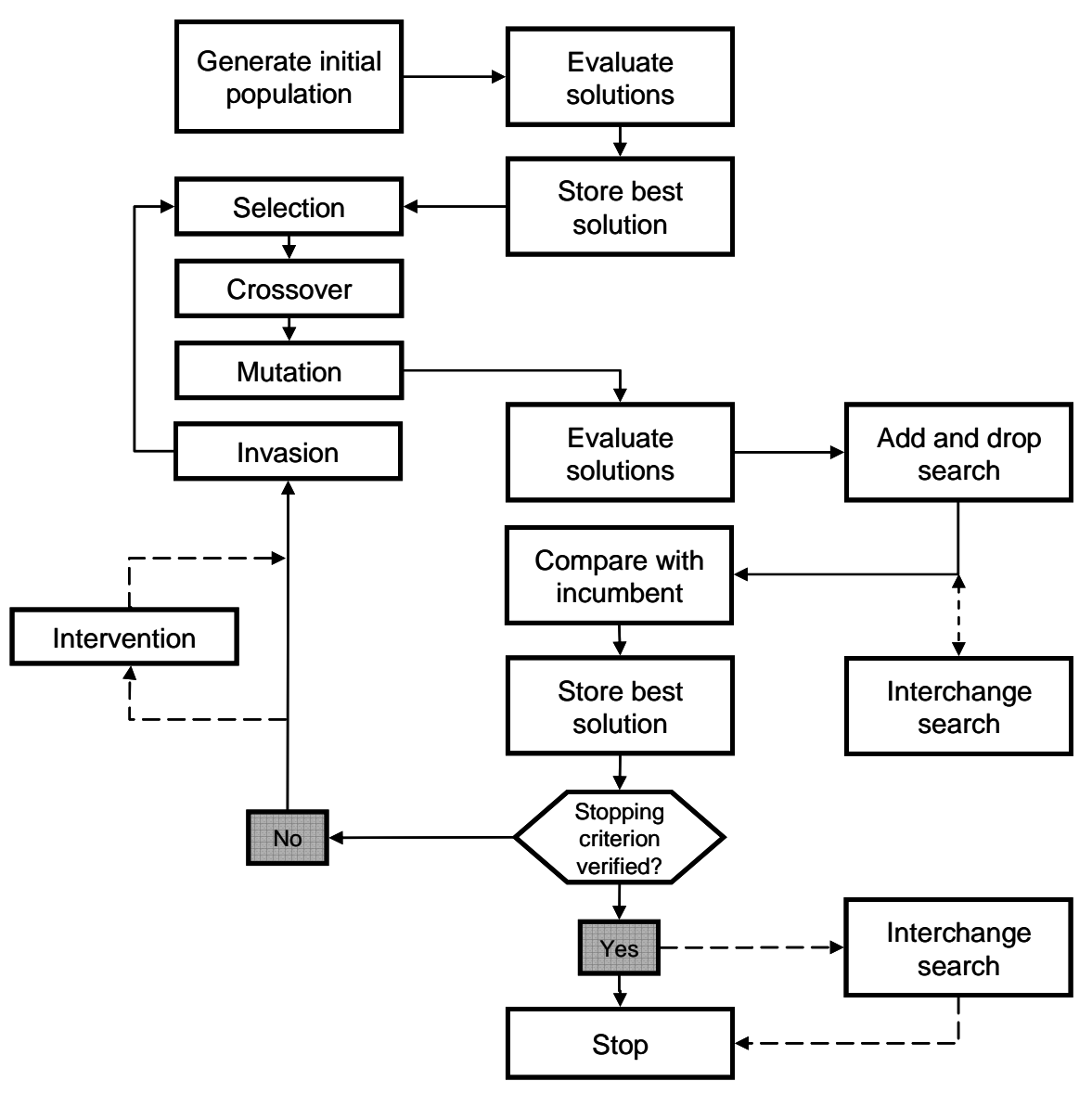

Figure 1 - Flowchart for the Enhanced Genetic Algorithm 
Network 1

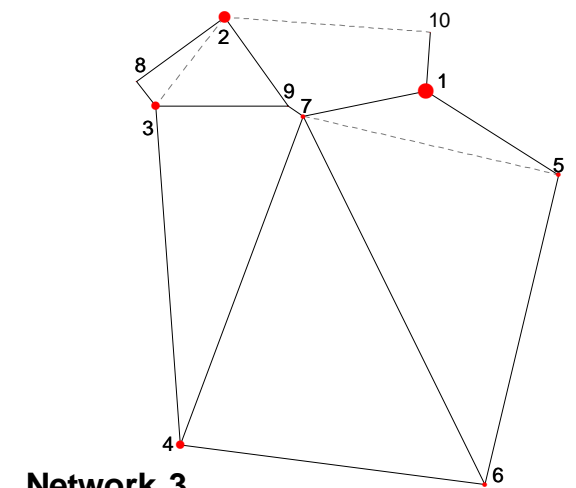

Network 3

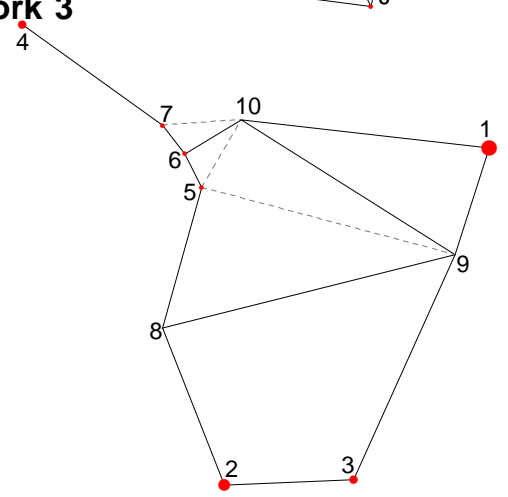

Network 2

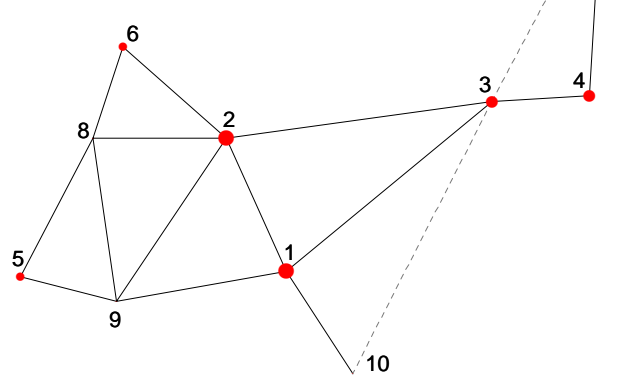

Population Centers

- 80000 inhabitants

- 150000 inhabitants

- 250000 inhabitants

- 400000 inhabitants

- 650000 inhabitants

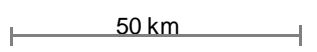

\section{Road Type}

Possible Road

Slow 2-lane highway

Figure 2 - Random networks 
Network 1

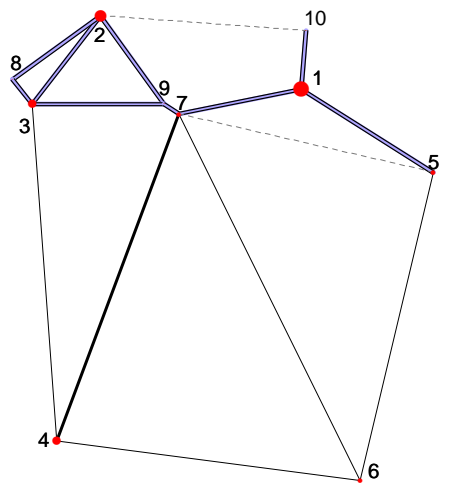

Network 3

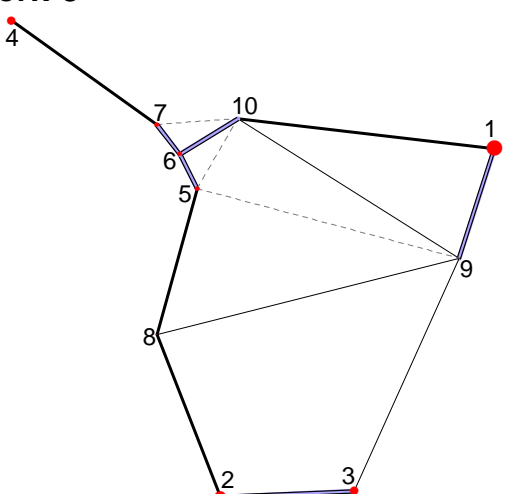

Network 2

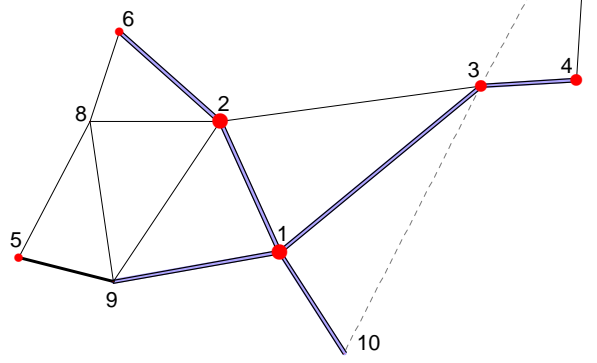

Road Type

Possible Road

Slow 2-lane highway

- Fast 2-lane highway

- 4-lane freeway

- 6-lane freeway

Figure 3 - Results for accessibility maximization 
Network 1

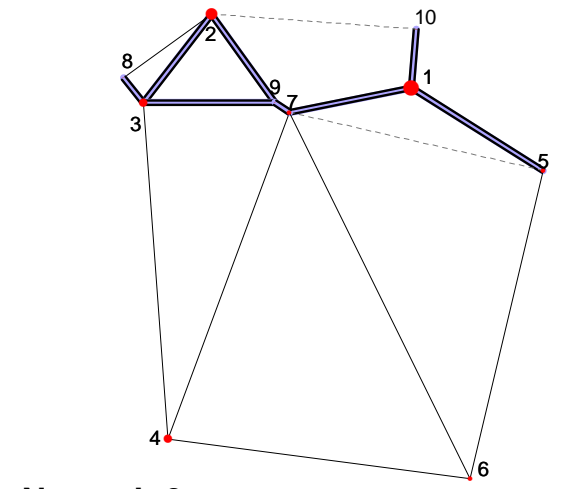

Network 3

4

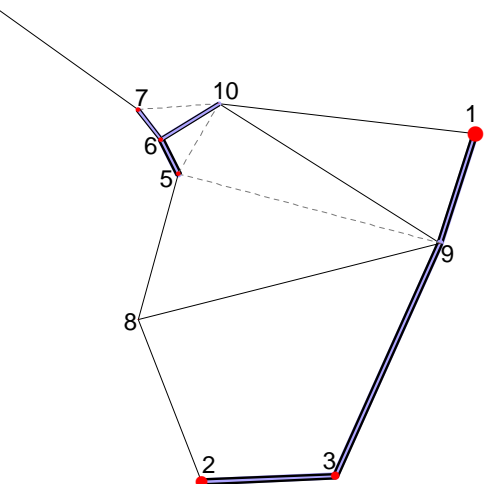

Network 2

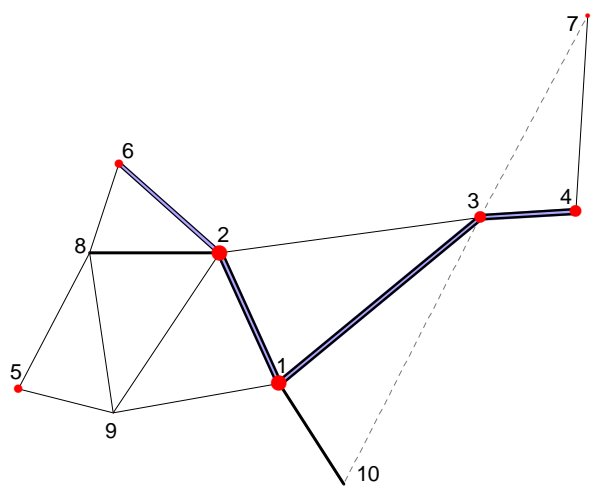

Road Type

Possible Road

Slow 2-lane highway

- Fast 2-lane highway

- 4-lane freeway

- 6-lane freeway

Figure 4 - Results for accessibility and spare capacity maximization 
Network 1

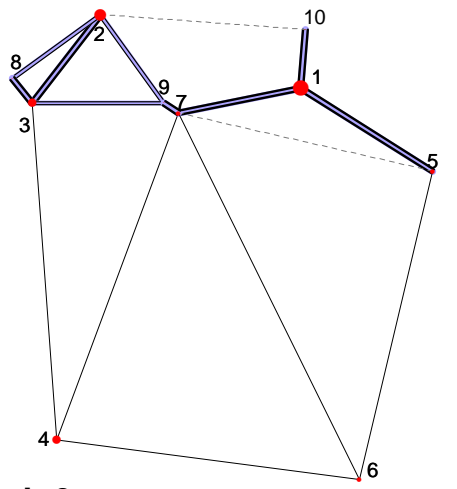

Network 3

4

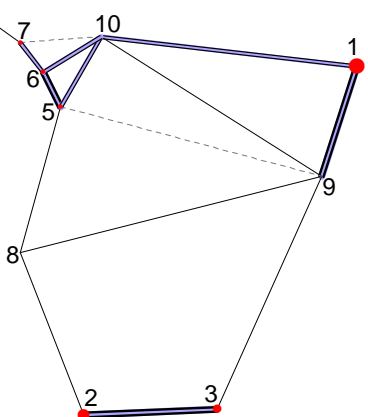

Network 2

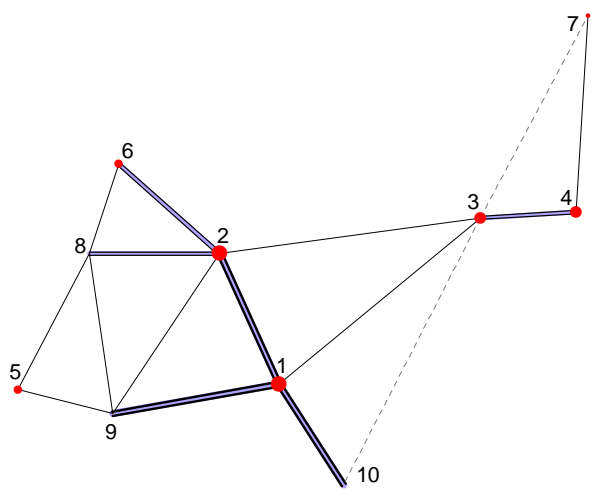

Road Type

Possible Road

Slow 2-lane highway

- Fast 2-lane highway

- 4-lane freeway

_ 6-lane freeway

Figure 5 - Results for accessibility and city evacuation capacity maximization 
Network 1

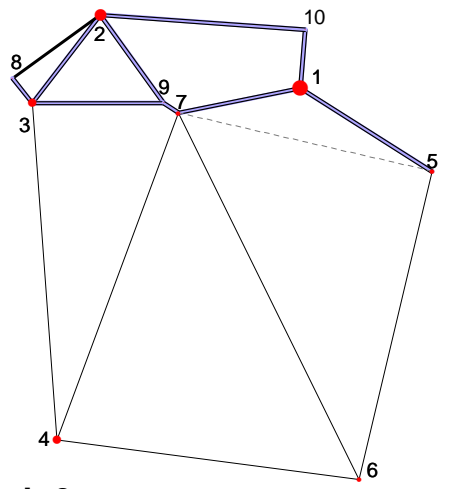

Network 3

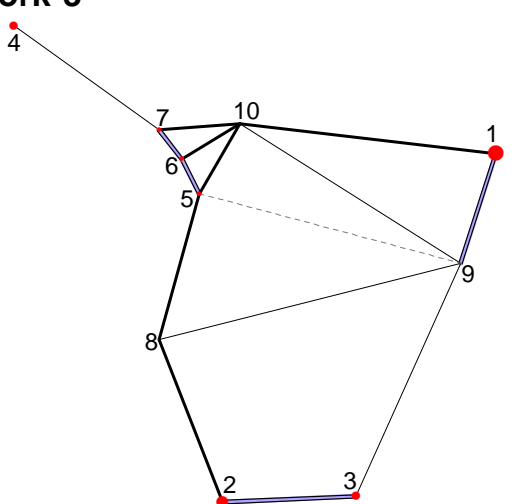

Network 2

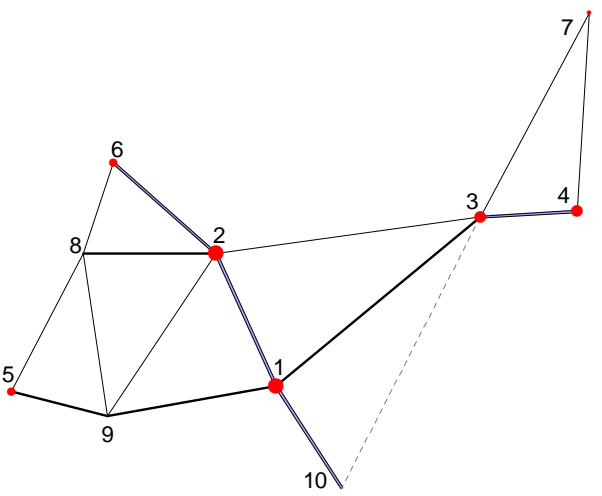

Road Type

Possible Road

Slow 2-lane highway

Fast 2-lane highway

_ 4-lane freeway

_ 6-lane freeway

Figure 6 - Results for accessibility maximization and network vulnerability minimization 
Table I - Road Construction and Upgrading Costs per Kilometer

\begin{tabular}{ccccc}
\hline \multirow{2}{*}{ From } & \multicolumn{3}{c}{ To } \\
\cline { 2 - 5 } & $\begin{array}{c}\text { Slow two-lane } \\
\text { highway }\end{array}$ & $\begin{array}{c}\text { Fast two-lane } \\
\text { highway }\end{array}$ & Four-lane freeway & Six-lane freeway \\
\hline Project Road & 1 & 2 & 3 & 4 \\
Slow two-lane highway & - & 1,5 & 2,5 & 3 \\
Fast two-lane highway & - & - & 2 & 2,5 \\
Four-lane freeway & - & - & - & 1 \\
\hline
\end{tabular}


Table II - Assessment Measures Variation for the Different Networks

\begin{tabular}{|c|c|c|c|c|c|}
\hline \multirow[b]{2}{*}{ Network } & \multirow[b]{2}{*}{ Assessment measure } & \multicolumn{4}{|c|}{ Objective(s) } \\
\hline & & $\begin{array}{l}\text { Accessibility } \\
\text { maximization }\end{array}$ & $\begin{array}{c}\text { Accessibility and } \\
\text { network spare } \\
\text { capacity maximization }\end{array}$ & $\begin{array}{l}\text { Accessibility and city } \\
\text { evacuation capacity } \\
\text { maximization }\end{array}$ & $\begin{array}{c}\text { Accessibility } \\
\text { maximization and } \\
\text { network vulnerability } \\
\text { minimization }\end{array}$ \\
\hline \multirow{4}{*}{1} & Accessibility & $47,7 \%$ & $45,1 \%$ & $46,7 \%$ & $46,9 \%$ \\
\hline & Weighted network spare capacity & $11,7 \%$ & $19,9 \%$ & $17,2 \%$ & $11,3 \%$ \\
\hline & Weighted city evactuation & $171,7 \%$ & $256,0 \%$ & $258,8 \%$ & $181,1 \%$ \\
\hline & Network vulnerability & $32,5 \%$ & $38,7 \%$ & $38,5 \%$ & $-75,5 \%$ \\
\hline \multirow{4}{*}{2} & Accessibility & $34,3 \%$ & $30,5 \%$ & $30,3 \%$ & $33,5 \%$ \\
\hline & Weighted network spare capacity & $10,1 \%$ & $14,3 \%$ & $12,6 \%$ & $8,1 \%$ \\
\hline & Weighted city evactuation & $113,1 \%$ & $126,6 \%$ & $152,9 \%$ & $83,2 \%$ \\
\hline & Network vulnerability & $37,2 \%$ & $37,3 \%$ & $106,6 \%$ & $-82,7 \%$ \\
\hline \multirow{4}{*}{3} & Accessibility & $35,3 \%$ & $30,4 \%$ & $32,3 \%$ & $34,5 \%$ \\
\hline & Weighted network spare capacity & $7,9 \%$ & $13,5 \%$ & $11,1 \%$ & $7,0 \%$ \\
\hline & Weighted city evactuation & $94,1 \%$ & $162,2 \%$ & $181,5 \%$ & $96,4 \%$ \\
\hline & Network vulnerability & $40,5 \%$ & $29,9 \%$ & $12,8 \%$ & $-78,2 \%$ \\
\hline
\end{tabular}

